\title{
Realizing 3D Spectral Imaging in the Far-Infrared: FIFI LS
}

\author{
L. W. Looney ${ }^{a}$, N. Geis ${ }^{a}$, R. Genzel ${ }^{a}$,W. K. Park ${ }^{a}$, A. Poglitsch $^{a}$, W. Raab ${ }^{a}$, \\ D. Rosenthal ${ }^{a}$, A. Urban ${ }^{a}$, and T. Henning ${ }^{b}$ \\ ${ }^{a}$ Max-Planck-Institut für Extraterrestrische Physik (MPE), Postfach 1603, \\ D-85740 Garching, Germany \\ ${ }^{b}$ Astronomisches Institut Universität Jena, Schillergäßchen 3, \\ D-07745 Jena, Germany
}

\begin{abstract}
We present a progress report on the design and construction of the Field-Imaging Far-Infrared Line Spectrometer (FIFI LS) for the SOFIA airborne observatory. The design of the instrument is driven by the goal of maximizing observing efficiency, especially for observations of faint, extragalactic objects. Thus, FIFI LS utilizes an integral field technique that uses slicer mirrors to optically re-arrange the two-dimensional field into a single slit for a long slit spectrometer. Effectively, a $5 \times 5$ pixel spatial field of view is imaged to a $25 \times 1$ pixel slit and dispersed to a 25 $\times 16$ pixel, two-dimensional detector array, providing diffraction-limited spatial and spectral multiplexing. In this manner, the instrument employs two parallel, medium resolution $(\mathrm{R} \sim 2000)$ grating spectrometers for simultaneous observations in two bands: a short wavelength band (42 to $110 \mu \mathrm{m}$ ) and a long wavelength band (110 to $210 \mu \mathrm{m})$. Overall, for each of the 25 spatial pixels, the instrument can cover a velocity range of $\sim 1500 \mathrm{~km} / \mathrm{s}$ around selected farinfrared lines with an estimated sensitivity of $2 \times 10^{-15} \mathrm{~W} \mathrm{~Hz}^{1 / 2}$ per pixel. This arrangement provides good spectral coverage with high responsivity. This paper does not include Figures due to astro-ph size limitations. Please download entire file at http://fifi-ls.mpe-garching.mpg.de/fifils.ps.gz.
\end{abstract}

Keywords: Integral Field Imaging, Spectrometer, Far-Infrared, grating, optical slicer, FIR, FIFI LS, FIFI, SOFIA

\section{INTRODUCTION}

Astronomers will soon have access to unprecedented spatial resolution and sensitivity in the far-infrared with the Stratospheric Observatory For Infrared Astronomy (SOFIA). Far-infrared astronomical observations, which are impossible from the ground due to water absorption, are necessary to understand fully a number of important astronomical problems and issues. Many astrophysical conditions require far-infrared probes because the areas of interest are mostly inaccessible at other wavelengths due to severe extinction from interstellar dust or the physics of interest is only manifest at far-infrared wavelengths. In particular, far-infrared spectroscopy, pioneered and developed on SOFIA's predecessor the Kuiper Airborne Observatory (KAO) and greatly extended with the Infrared Space Observatory (ISO), will provide an injection of important data into astrophysical issues.

Building upon the success of our previous imaging Fabry-Perot far-infrared spectrometer (FIFI国) that was designed for the KAO, Spectrometer (FIFI LS l $_{\text {) }}$ ). FIFI LS will utilize integral field spectral imaging in two wavelength bands: 42 to 110 $\mu \mathrm{m}$ and 110 to $210 \mu \mathrm{m}$. This will allow the instrument to simultaneously obtain dual wavelength band, spectral imaging of a $5 \times 5$ pixel field, without scanning a Fabry-Perot or multiple pointings with a long-slit spectrometer. Thus, FIFI LS is designed as a "major-step" forward and will take advantage of the unique benefits that SOFIA offers.

With the increased sensitivity and resolution provided by SOFIA, the main scientific targets for FIFI LS will include the detailed morphological studies of: (1) the heating and cooling of galaxies, (2) star formation and the interstellar matter under low-metalicity conditions, as found in dwarf galaxies, (3) active galactic nuclei and their environment, (4) merging and interacting galaxies, and (5) large surveys of nearby galaxies. To reach our scientific goals, very high observing sensitivities and efficiencies are essential, requiring a compromise of spectral resolution; however, for the science objectives listed above, a comparably low spectral resolution $(\mathrm{R} \sim 2000)$ is more than sufficient. Overall, FIFI LS on SOFIA will be more sensitive than the ISO Long-Wavelength Spectrometer and have much higher spatial resolution and mapping capabilities. As a future option, an extension of the instrument to the 25-42 $\mu \mathrm{m}$ range is planned upon availability of the Si:Sb detector arrays developed for SIRTF.

email: lwl@mpe.mpg.de; http://fifi-ls.mpe-garching.mpg.de 
Figure 1. Focal plane projections of the long and short wavelength channels of FIFI LS, showing the instantaneous field of view for both channels, the re-arrangement of the $5 \times 5$ field of view onto a $25 \times 1$ slit, and the spectral projection of the integral field onto the $16 \times 25$ detector arrays.

Figure 2. Solid model of the FIFI LS optical system, viewed from the top. The IR light enters from the left and is split by a diachronic into the long and short spectrometers - the upper is for short $\lambda$ and the lower is for long $\lambda$. The $16 \times 25$ detector arrays are the biconic surfaces, second from the right.

\section{INSTRUMENT DESIGN}

\subsection{Spectrometer Concept}

FIFI LS achieves 2-dimensional spatial mapping and simultaneous spectral multiplexing by optically slicing, or rearranging, the 2D field of view onto a single slit, which is then dispersed via a standard long-slit spectrometer. This type of "optical slicer" was originally devised for laboratory spectroscopy and later successfully implemented for near-infrared astronomy, 0 but this is the first time that this technique has been applied in the far-infrared. Specifically, FIFI LS has a $5 \times 5$ pixel field of view which is sliced into five individual slitlets, re-arranged into a continuous $25 \times 1$ pixel slit, and finally, fed into a grating spectrometer and dispersed onto a $2 \mathrm{D} 16 \times 25$ pixel detector array.

One of the other unique aspects of FIFI LS is its dual-channel nature. By using a dichroic beamsplitter in the entrance optics and two optical slicers, FIFI LS covers the diffraction-limited field of view simultaneously in two wavelength bands: a short wavelength band 42 to $110 \mu \mathrm{m}$ and a long wavelength band 110 to $210 \mu \mathrm{m}$. Fig. 1 shows how the dual channel integral field system is realized in more detail as a projection of the focal plane onto the two detector arrays with diffraction-limited optics. On the right side of the figure is the long wavelength channel of the spectrometer which has a pixel scale of $14^{\prime \prime}$ per pixel. On the left side of the figure is the short wavelength channel which has a pixel scale of $7^{\prime \prime}$ per pixel. This of course implies that the shorter wavelength channel has a smaller field of view, as demonstrated in Fig. 1. Overall, this scheme ensures that for all spatial elements in the field, spectra are observed simultaneously in the two bands, thereby increasing observing efficency.

\subsection{Optical Design}

The geometrical layout, optimization, and analysis of the entire spectrometer optics was carried out with the optical design software ZEEMAX-EE Ver. 8.0. The two spectrometers were designed to yield diffraction-limited image quality: the geometrical spot diameters are small compared to the diffraction disk diameter of a point source imaged by the telescope. A 3-D solid model of the complete FIFI LS optics is shown in Fig. 2, viewed from the top; all optical components are cooled to cryogenic temperatures.

A rotating K-mirror assembly (hidden on the left in Fig. 2) at liquid nitrogen temperature compensates for rotation of the field of view during long integration times. Two chopped calibrator sources with temperatures close to liquid nitrogen can be switched into the beam for internal calibration and flat-fielding at irradiation levels close to that of the SOFIA telescope background. The calibrator sources are located near a pupil image so that the light paths are equivalent for internal calibration and observation. All optics after the calibration system are at liquid helium temperature. After the calibration optics, the beam enters the spectrograph through a cold Lyot stop (not shown in Fig. 2) to suppress light diffracted by the entrance aperture. A far-infrared dichroic beam splitter feeds both the long and short wavelength spectrometer. In the short wavelength branch, re-imaging optics double the image size on the image slicer.

In Figure 3, the optical slicer is shown in more detail. The light enters into the slicer system from the flat mirror (upper-left in Fig. 3), then continues down toward the slicer mirrors (the cube-like object near the left bottom of Fig. 3). These 5 mirrors in the mirror stack form the image slicer, which acts as a field mirror creating a pupil image on one of the 5 re-imaging capture mirrors (the five mirrors near the top-middle). The capture mirrors re-arrange 
Figure 3. A 3D model of the optical slicer assembly. Light enters the slicer system from the upper-left field mirror, and is deflected down toward the lowest surface, the slicer mirrors. The five slicer mirrors re-image the pupil onto the 5 separated capture mirrors, which is then focused onto the slit mirrors that are at the center of the image, and final into the spectrometer. In the background is a grating and collimator mirror.

Figure 4. Efficiency of the gratings as a function of wavelength and diffraction order. The insets show the grating profiles: short $\lambda$ grating on the left and long $\lambda$ grating on the right (not to scale).

the images of the 5 slices along one, slightly curved line on the slit-mirror, near the center of the image. Working in combination, these three mirrors, the slicer, capture, and slit mirrors, perform the slicing and re-arranging of the field of view onto the entrance slit for a grating spectrograph. In addition, the three mirror system re-aligns the pupil of each slit, so that the virtual pupils of each slice coincide. In this case, we do not use simple flat mirrors for the slicing process, as is usually done in near-infrared instruments, because of the larger $\mathrm{A} \times \Omega$ product (area times the beam solid angle); curved mirrors allow for a much more compact slicer assembly design.

The FIFI LS spectrometers use slightly off-axis $\left(0.5^{\circ}\right)$ Littrow mounted gratings (the long rectangular optics in Fig. 2); a truly Littrow mounted grating spectrometer would have the same entrance and exit optical paths, but in the FIFI LS system the entrance and exit paths are slightly separated so that the outgoing beam can easily be re-imaged onto the detectors. This arrangement allows for the dual use of the two anamorphic collimators (the two large mirrors right-most and the two large mirrors on top and bottom in Fig. 2). From the image slicer, the beam continues in each channel via the anamorphic optics, which expand the beam to an elliptical shaped cross-section that illuminates the grating over its $300 \mathrm{~mm}$ length, attaining the proposed spectral resolution of about 1000-2000 $(\Delta \nu \sim 100-250 \mathrm{~km} / \mathrm{s})$ In each return path, the anamorphic re-imaging is used to match the spatial and spectral resolution of the system to the square pixels of the detector.

In order to cover the wide wavelength range necessary, the Littrow-mounted gratings are operated in the 1st order (long wavelengths) or 1st and 2nd orders (short wavelengths). To select first or second diffraction order, exchangeable filters are utilized in the short-wavelength branch of the spectrometer. The observing wavelength of each spectrograph is then tuned by tilting the grating, and exchangeable filters are used to select the appropriate working order of the grating.

Since the grating efficiency strongly depends upon wavelength and diffraction order, the groove profile and separation for each grating were optimized separately. Rigorous vectorial diffraction analysis of the grating efficiency in the actual Littrow configuration were performed using the PCGrate-1E Ver. 3.0 software. The calculated grating efficiencies as a function of wavelength and diffraction order are shown in Fig. 4. Our goal was to maximize the efficiencies across the two bands. For the long wavelengths, this was done by using a symmetric grating profile that provided a high efficiency across the band, and for the shorter wavelengths one grating order was insufficient, and the best result was obtained by maximizing the efficency over two orders by using an asymmetric grating profile. In general, the optical parameters of FIFI LS are summarized in Table 1.

\subsection{Grating Mechanical Layout}

To cover the specified wavelength range of 42 to $110 \mu \mathrm{m}$ and 110 to $210 \mu \mathrm{m}$ in first and second order, both gratings have to be tilted by an angle of $\pm 20^{\circ}$. However, to reach a spectral accuracy of $\sim 100-250 \mathrm{~km} / \mathrm{s}$, the grating has to be moved and controlled with a precision of less than $4^{\prime \prime}$ while maintaining mechanical stability of the optical surface. In addition, this precision must be reached at liquid helium temperatures. In order to minimize deformation to the grating surface from vibration, the grating structure design was extensively tested with finite element modeling. The final grating structure, as shown in Fig. 5, has $<1 \mu \mathrm{m}$ of deflection with $10 \mathrm{~N}$ of applied force at the outer edge of the structure.

To obtain a large tilting range and precise positioning, the grating is actuated by a two stage tilting mechanism. The first stage, for coarse positioning, consists of a support structure connected to the bottom of the grating. The 


\begin{tabular}{lcc}
\hline & Short $\lambda$ Spectrometer & Long $\lambda$ Spectrometer \\
\hline Wavelength Range & $42-110 \mu \mathrm{m}$ & $110-210 \mu \mathrm{m}$ \\
Field of View & $35^{\prime \prime} \times 35^{\prime \prime}$ & $70^{\prime \prime} \times 70^{\prime \prime}$ \\
Num. of Spatial Pix. & $5 \times 5$ & $5 \times 5$ \\
Num. of Spectral Pix. & 16 & 16 \\
Pixel size & $3.6 \times 3.6 \mathrm{~mm}$ & $3.6 \times 3.6 \mathrm{~mm}$ \\
f/D at collimator & 20 & 10 \\
f/D on detec. array & 48 & 24 \\
Collim. beam dia. & $40 \times 80 \mathrm{~mm}$ & $80 \times 160 \mathrm{~mm}$ \\
Grating lines & $12 / \mathrm{mm}$ & $8.5 / \mathrm{mm}$ \\
Grating angle & $28-68^{\circ}$ & $28-68^{\circ}$ \\
Groove profile & asymmetric & symmetric \\
Groove separation & $83 \mu \mathrm{m}$ & $118 \mu \mathrm{m}$ \\
Groove depth & $42 \mu \mathrm{m}$ & $140 \mu \mathrm{m}$ \\
Enclosed angle & $84^{\circ}$ & $44^{\circ}$ \\
Resolution $(\mathrm{c} \Delta \lambda / \lambda)$ & $100-250 \mathrm{~km} / \mathrm{s}$ & $100-250 \mathrm{~km} / \mathrm{s}$ \\
Instantaneous Vel. coverage & $1300-3000 \mathrm{~km} / \mathrm{s}$ & $1300-3000 \mathrm{~km} / \mathrm{s}$ \\
\hline
\end{tabular}

Table 1. Optical Parameters of FIFI LS.

Figure 5. 3D model of the diffraction grating.

support structure is driven by a roller-screw lever arm mechanism (a so-called sine-bar mechanism) that tilts the grating via a push-pull movement. The roller screw is driven by a stepper motor at liquid nitrogen temperature that is thermally isolated from the grating by a magnetic feed-thru. The second stage, for fine positioning, utilizes a PZT, which drives the grating with respect to the support structure via a directly attached lever arm. The PZT movement is controlled in a software loop with closed-loop bandwidth up to $1 \mathrm{kHz}$. Additionally, an eddy current damping system is mounted to the grating to minimize in-flight vibrations of the airplane, which are a perpetual source of error on airborne experiments, especially affecting moving parts where resonant modes can raise error motion to an unacceptable value.

\subsection{Grating Position Read-out}

For highly reliable measurement of the angular position of the gratings, we directly attached an INDUCTOSYN ${ }^{T M}$ position transducer. An INDUCTOSYN ${ }^{T M}$ is effectively a transformer with the primary and secondary windings placed on a rotor and stator, respectively. The winding pattern on the rotor is excited by a $10 \mathrm{kHz}$ signal, while on the stator, there are two periodic patterns that are $90^{\circ}$ out of phase with each other. In operation, the rotor and stator windings inductively couple such that the two output signals from the stator have amplitudes which vary as the sine and cosine functions based on the relative position in each winding cycle. By comparing the two amplitudes, a high-resolution difference positional accuracy is obtained.

The position transducers work with a fairly high operating current of $\sim 0.25$ Amperes. To provide thermal insulation of the $4 \mathrm{~K}$ worksurface, only thin signal wires are tolerated within the cryogenic regions. Thin wires, on the other hand, raise the power dissipation, leading again to higher heat input. To overcome this problem, we use a superconducting transformer which steps down the signal voltage by a factor of 50 after entering the $4 \mathrm{~K}$ region. In this way, a high-voltage, but low- current excitation signal, can be conveyed via thin wires, reducing dissipation losses. 
Figure 6. Solid model of the FIFI LS system. FIFI LS is mounted to the SOFIA instrument flange on the right side. This image shows the three cryogenic containers (top-most), the optical worksurfaces (hanging from the cryogenic containers via G-10 tabs, colored black), the optics, and the guiding camera (bottom-most).

Figure 7. Solid model of the three cryogenic containers, from top to bottom: liquid nitrogen, liquid helium, and the smaller superfluid helium.

The relative amplitudes of the two transducer outputs are a measure of the actual position of the grating, with proper initial calibration. Both signals are amplified separately in a low-noise amplifier stage and translated into 14 bit digital position data by a Resolver-to-Digital Converter. Since the transducer output produces a roll over of the position data every $1.4^{\circ}$, an additional loop counter keeps track on the position data over the full $40^{\circ}$ tilting range of the grating. First tests with a prototype setup at liquid helium temperature, showed that an angular resolution of less than $0.5^{\prime \prime}$ can be obtained easily.

\subsection{Detectors}

As meptioned above, FIFI LS uses two $16 \times 25$ detector arrays to cover the $42-110 \mu \mathrm{m}$ and $110-210 \mu \mathrm{m}$ wavelength bands We chose to use Gallium-doped Germanium photoconductor detectors since they are proven to be very sensitive in the wavelength range $40-120 \mu \mathrm{m}$, and, with the application of $\sim 600 \mathrm{~N} \mathrm{~mm}^{-2}$ of stress, their wavelength sensitivity shifts to $100-220 \mu \mathrm{m}$. our design and initial testing is discussed in detail in this volume.

\section{CRYOSTAT}

The FIFI LS instrument is directly attached to the science instrument mounting flange of the SOFIA telescope. In Fig. 6, a 3D rendered model of the FIFI LS instrument is shown. The cryostat is enclosed by a vacuum vessel which also provides the mechanical interface for mounting. Mounted to the bottom of the vacuum vessel are the dichroic beam splitter and the field optics for the focal plane guiding camera. The dichroic filter separates the telescope's light (entering Fig. 6 on the bottom right) into two beams: the optical, directed downward to the telescope guiding camera, and the infrared, directed upward into the cryostat. The infrared beam from the telescope enters the vacuum vessel through a polyethylene window, which also serves as a pressure barrier between stratospheric pressure and the vacuum inside the instrument.

There are three cryogenic containers in FIFI LS for liquid nitrogen, liquid helium, and superfluid helium. The manner in which the three vessels are mounted together is shown in Fig. 7, a center cut-thru the cryogenic vessels. The liquid nitrogen container has a capacity of 25 liters, which provides cooling for the outer radiation shields, the liquid nitrogen worksurface and the entrance optics (i.e. the K-mirror assembly and the re-imaging optics). The cut-thru of the liquid nitrogen vessel in Fig. 7 shows two of its inner support ribs. The liquid nitrogen worksurface is suspended from the warm vacuum vessel by G-10 fiberglass stand-offs which provide high mechanical stiffness and low thermal conductivity. The liquid nitrogen container is designed for a cryogen holding time of about 28 hours.

The 35 liter main liquid helium reservoir provides cooling for the inner radiation shields and the liquid helium optical bench. The liquid helium optical bench, suspended from the liquid nitrogen worksurface by G-10 tabs, mechanically supports and cools all of the optical components after the calibration system (excluding the detectors). The expected cryogen holding time for the main liquid helium reservoir is up to 50 hours.

Since the detector arrays require operating temperatures below $4 \mathrm{~K}$ they are mounted to a small 2.8 superfluid helium tank, which is again suspended by G-10 tabs from the liquid helium optical bench. This tank is pumped in order to reach a temperature of $\sim 2 \mathrm{~K}$. The expected maximum holding time for the liquid helium tank is about 18 hours. To ensure safe operation during flight, all cryogen vessels are provided with coaxial neck tubes and warm pressure relief valves. 
Figure 8. Finite element analysis of the vacuum vessel, $\mathrm{LN}_{2}$ vessel, and the LHe vessel under 3.5 bars. The distortion is overemphasized, never exceeding $1 \mathrm{~mm}$ of displacement.

\begin{tabular}{lccc}
\hline Wavelength Range & & $42-110 \mu \mathrm{m}$ & $110-210 \mu \mathrm{m}$ \\
\hline Pixel size & & $7^{\prime \prime} \times 7^{\prime \prime}$ & $14^{\prime \prime} \times 14^{\prime \prime}$ \\
Field of view $5 \times 5$ pixels & & $35^{\prime \prime} \times 35^{\prime \prime}$ & $70^{\prime \prime} \times 70^{\prime \prime}$ \\
Resolution $(\mathrm{c} \Delta \lambda / \lambda)$ & $100-250 \mathrm{~km} / \mathrm{s}$ & $100-250 \mathrm{~km} / \mathrm{s}$ \\
Instantaneous Vel. coverage & & $1300-3000 \mathrm{~km} / \mathrm{s}$ & $1300-3000 \mathrm{~km} / \mathrm{s}$ \\
Point source det. limit & $\lambda=50 \mu \mathrm{m}$ & $5.5 \times 10^{-17} \mathrm{~W} / \mathrm{m}^{2}$ & $\ldots .$. \\
$(5 \sigma$ in $1 \mathrm{hr})$ & $\lambda=100 \mu \mathrm{m}$ & $3.5 \times 10^{-17} \mathrm{~W} / \mathrm{m}^{2}$ & $\ldots .$. \\
& $\lambda=150 \mu \mathrm{m}$ & $\ldots .$. & $2.2 \times 10^{-17} \mathrm{~W} / \mathrm{m}^{2}$ \\
& $\lambda=200 \mu \mathrm{m}$ & $\ldots .$. & $1.4 \times 10^{-17} \mathrm{~W} / \mathrm{m}^{2}$ \\
\hline
\end{tabular}

Table 2. FIFI LS performance specifications.

\subsection{Cryostat Analysis}

In order to comply with Federal Aviation Administration requirements, we are designing the Cryostat to withstand 3 times the operational pressure - about 3.5 bars. Fig. 8 shows finite element analysis results for the three large vessels at 3.5 bars of internal pressure- overemphasizing the deformation for display purposes The maximum deformation in the three vessels are $0.91 \mathrm{~mm}$ for the vacuum vessel, $0.20 \mathrm{~mm}$ for the $\mathrm{LN}_{2}$ vessel, and $0.24 \mathrm{~mm}$ for the LHe vessel. In addition, we used FEA for determining the best design for stability of the internal optical work surfaces and to minimize the amount of stress on the welding joints of the vessels. In the latter case, for example, we find that the best approach is to create a nearby zone that can elastically deform, thereby relocating areas of high stress away from the welding seam.

\section{SCIENTIFIC CAPABILITIES}

FIFI LS employs two fixed pixel sizes of $7^{\prime \prime}$ (short wavelength spectrometer) and 14" (long wavelength spectrometer), respectively, determined by the image slicer. The $5 \times 5$ pixel fields of view are observed simultaneously with two Ge:Ga photoconductor arrays. Observing wavelength are adjusted by tilting the Littrow mounted grating in each channel. Spectral coverage of $\sim 1500 \mathrm{~km} / \mathrm{s}$ around a selected far-infrared line is obtained simultaneously for all 25 spatial pixels. A summary of important instrument properties is shown in Table 2.

\section{REFERENCES}

1. A. Poglitsch, J.W. Beeman, N. Geis, R. Genzel, M.H.E.E. Haller, J. Jackson, M. Rumitz, G.J. Stacey, and C.H. Townes, Int J. IR 83 Millimeter Waves, 12, p. 859 (1991).

2. N. Geis, R. Genzel, M. Haggerty, F. Herrmann, J. Jackson, S.C. Madden, T. Ncola, A. Poglitsch, M. Rumitz, G.J. Stacey, R. Timmermann, and C. H. Townes, ASP Conference Series 73, Airborne Astronomy Symposium on the Galactic Ecosystem, M.R. Haas,J.A. Davidson, and E.F. Erickson eds., p. 547 (1995).

3. N. Geis, A. Poglitsch, W. Raab, D. Rosenthal, G. Kettenring, T. Henning, \& J.W. Beeman, SPIE Proc. Infrared Astronomical Instrumentation, Fowler, A.M. eds., p. 973 (1998).

4. W. Raab, N. Geis, L. Looney, A. Poglitsch, D. Rosenthal, A. Urban, T. Henning, \& J.W. Beeman, SPIE Proc. 3759 Infrared Spaceborne Remote Sensing VII, Andersen, B. eds., p. 86 (1999)

5. W. Benesch \& J. Strong, J. Opt. Soc. Am. 41, p. 252 (1951).

6. A. Krabbe, L. Weitzel, H. Kroker, L. E. Tacconi-Garman, M. Cameron, N. Thatte, G. Samann, T. Boeker, R. Genzel, \& . Drapatz, Proc. SPIE 2475, Infrared Detectors and Instrumentation for Astronomy, A. M. Fowler, ed., p. 172, (1995). 
7. D. Rosenthal, J.W. Beeman, N. Geis, L. Looney, A. Poglitsch, W.K. Park, W. Raab,, \& A. Urban, Proc. SPIE 4014, This volume (2000)

8. G.J. Stacey, J.W. Beeman, E.E. Haller, N. Geis, A. Poglitsch, \& M. Rumitz, Int. J. Inf. Mill. Wave, 13, 1689 (1992)

9. J.W. Beeman \& E.E. Haller, Inf. Phys. Tech., 35, 827 (1994) 